\title{
Design of the Counting System for the Sewing Machine Production Line
}

\author{
Zhihong Xiao \\ School of Electronic Engineering \\ Xi'an Shiyou University \\ Xi'an, China \\ e-mail:xzhong@xsyu.edu.cn
}

\begin{abstract}
A counting system of the sewing machine production line is introduced. The circuit and software design of the system are also given. With AT89C51 used as the control center and optoelectronic switch used as the sensor, the system can detect actual quantity of the line, display the time and plan production. The experimental results show that the system has the advantages of good reliability, easy operation and high accuracy. It can be widely used in the field of home appliance and food manufacturing.
\end{abstract}

Keywords-production line; counting system; AT89S51; optoelectronic switch component

\section{INTRODUCTION}

With the development of modern production, the traditional artificial measurement methods of counting product quantity are not accurate. In order to manage and count parts or finished products on automated production line timely and accurately, the automatic detection and counting devices are needed to install .The automatic devices can display the number of products and provide dynamic data to the computer management system timely, which are the basis of process control[1-4].

This system is designed for the sewing machine factory assembly line. There are thirty sewing machine production lines in the factory. Each line needs the counting system. The system must have many functions such as detecting and displaying the product quantity of the production line realtime, displaying the time and plan production data, setting parameter online, transmitting data to the master control room, etc.

\section{THE COMPOSITION OF THE HARDWARE SYSTEM}

The system adopts AT89S51 as the core controller, which is composed of keyboard circuit, signal detection circuit, watchdog circuit, clock circuit, display circuit and communication circuit. System diagram is shown in Fig.1[56].

AT89S51 is a low-power, high-performance CMOS 8-bit microcontroller with $4 \mathrm{~KB}$ of ISP flash memory. The device uses Atmel high-density, nonvolatile memory technology and is compatible with the industry-standard 80C51 instruction set and pinout. On-chip flash allows program memory to be reprogrammed in-system or by a conventional nonvolatile memory programmer. The system clock uses 6 $\mathrm{MHz}$, port 0 is data port, $\mathrm{P} 1.1 \sim \mathrm{P} 1.6$ are connected to the keyboard circuit, port 2 is used to address control, P3.3, P3.4,
P3.5 joint signal detection circuit, P1.0 is connected to watchdog reset circuit and to provide a periodic pulse input signal.

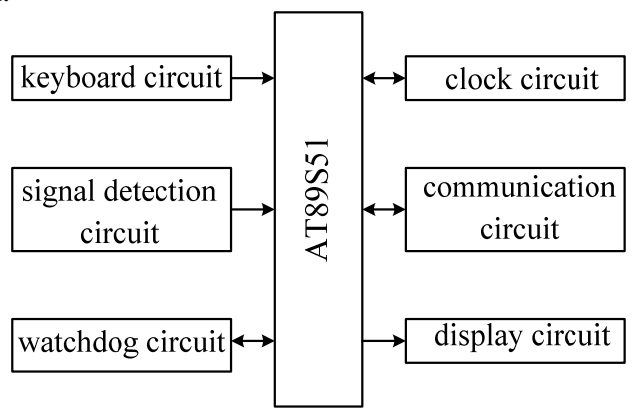

Figure 1. System diagram

Signal detection circuit is used to test product quantity of production line and provide corresponding number of pulse signal to microcomputer, when products pass through the production line. AT89S51 uses on-chip timing / counter to count the number of products. Each product passes forward, the counter "add 1 ".

The display needs to show the current time, plan production, actual production, etc. The current time is generated by the clock chip DS12887. The plan production which is inputted by the keyboard is the production numbers that needed to get in all day. Actual production is the actual numbers counted by the circuit. The system uses serial communication, and SCM connects to PC through the MAX485.

\section{A. Watchdog Reset Circuit}

The watchdog reset circuit uses MicroMonitor Chip DS1232 which is the produce of American Dallas Company. It has many advantages such as higher safe reliability, lower price, better anti-interference ability, etc. The DS1232 monitors three vital conditions for a microprocessor: power supply, software execution, and external override. First, a precision temperature-compensated reference and comparator circuit monitors the status of $\mathrm{V}_{\mathrm{CC}}$. When an outof-tolerance condition occurs, an internal power fail signal is generated which forces reset to the active state. When $V_{C C}$ returns to an in-tolerance condition, the reset signals are kept in the active state for a minimum of $250 \mathrm{~ms}$ to allow the power supply and processor to stabilize. Second, the DS1232 performs pushbutton reset control. The DS1232 bounces the pushbutton input and guarantees an active reset 
pulse width of $250 \mathrm{~ms}$ minimum. The third function is a watchdog timer. The DS1232 has an internal timer that forces the reset signals to the active state if the strobe input is not driven low prior to time-out. The watchdog timer function can be set to operate on time-out settings of approximately $150 \mathrm{~ms}, 600 \mathrm{~ms}$, and 1.2 seconds. The function is very effective to prevent the interference caused by the microprocessor crash. It forces $R S T$ and $\overline{R S T}$ signals to the active state when the ST input is not stimulated for a predetermined time period. In the system the time period is set by the TD input to be typically $150 \mathrm{~ms}$ with TD connected to ground. P1.0 is connected to ST to provide periodic input signal. $\overline{R S T}$ is connected to the INT0 of the microcomputer[7].

\section{B. Clock Circuit}

The clock circuit uses a 8-bit parallel interface real time clock / calendar chip DS12887, which contains a lithium battery power to run for more than 10 years without losing data. There is 12 hours system or 24 hours system for a day .Two kinds of bus timing mode can be selected in this clock chip such as Motorola and Intel[8-9]. AT89S51 and DS12887 interface circuit is shown in Fig.2.



Figure 2. AT89S51 and DS12887 interface circuit

\section{The Keyboard and Display Circuit}

The display circuit is composed of 16 common anode LED digital tubes and its driving circuit. The plan production and actual production are displayed by 4 digital tubes and the current time is displayed by 12 digital tubes. Digital tubes are driven by CD4511 with latch function. The data signals of CD4511 are given by SCM P0. The chip select signal of CD4511 is given by decoder. The driver circuit of two digital tubes is shown in Fig. 3.

The keyboard circuit's function is to determine the working state of the system and set parameters. There are two kinds of working state of the circuit, one kind is set condition, and the other one is running state. In the set condition we can set plan production and correct the current time parameters by keyboard. In the running state, the system can count product quantity of production line, and can correct the actual production online at the same time.
There is dithering elimination circuit composed of resistance-capacitance circuit, which is used to relieve interference of key dithering.

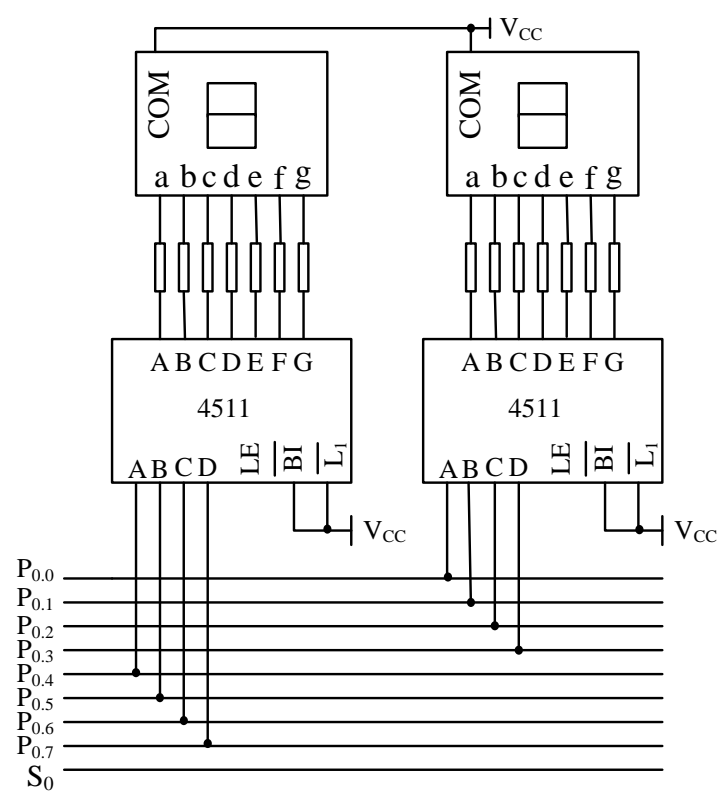

Figure 3. Driver circuit of two digital tubes

\section{Signal Detection Circuit}

The signal detection circuit is the key of the whole system. We must set detection point in the right position on the automatic production line, and then test the products quantity. The count methods consist of contact measurement and non-contact measurement. But in most cases, the products on the assembly line are difficult to determine the position. The contact detection mode is difficult to achieve, and non-contact detection has many advantages. This system adopts BR85-BP-ST7X/E photoelectric switch sensor, in which the transmitter and the receiver are integrated into a whole. Photoelectric switch adopts photoelectric element as detecting element, and the transmitter emits light through the mirror reflected back to the receiver, when a object is detected by the light, the photoelectric switch produces a detection switch signal[10-11].

The signal detection circuit is shown in Fig.4. Its task is to test the product quantity on production line. IN0, IN1 is photoelectric switch output signal, low level effective. After shaping, filtering the signal by the circuit, they can be send into microcomputer to count. The circuit should be able to judge the direction of the operation of the product, because problem products should be back to retest in the production line. When the product pass forward, counter "plus $1 "$. When the product pass backward, counter "minus 1". To achieve this function, two photoelectric switches are installed on the production line: they can determine product operation direction according to the order of the effective signal into single chip microcomputer. 


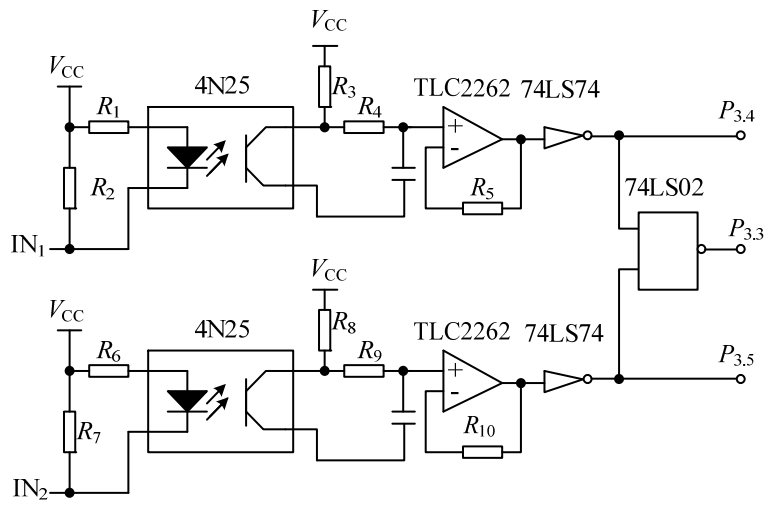

Figure 4. Signal detection circuit

\section{SYSTEM SOFTWARE DESIGN}

The system software includes a main program, data acquisition subprogram, key processing subprogram, interrupt subprogram, showing subprogram and communication subprogram, etc. The main program mainly complete device initialization, determine whether the key is pressed and whether the interrupt request exists, and then, according to the result of judgment calls the corresponding subroutine. The flow chart of main program is shown in Fig. 5 .

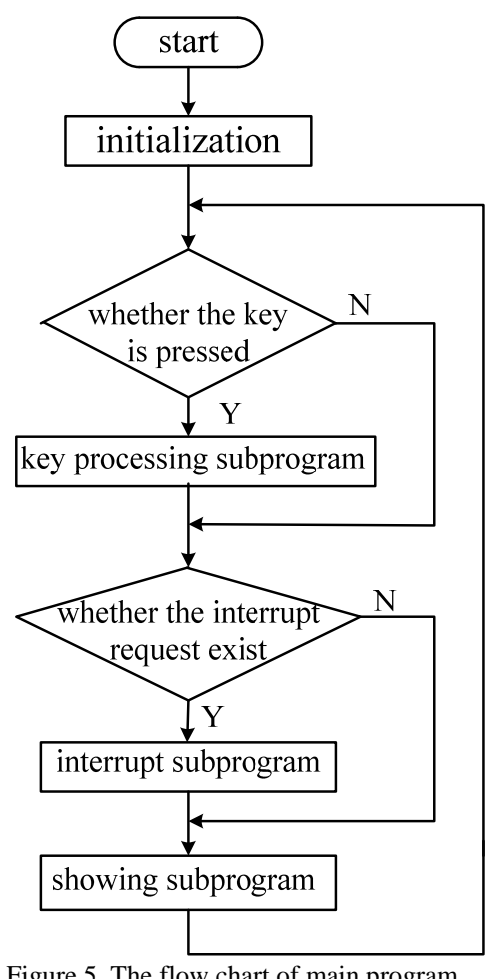

\section{CONCLUSION}

The put forward counting system was installed to measure the product quantity on production line of a Sewing Machine plant. With commissioning being completed, the system was tested. The experimental results are shown in table 1.

TABLE 1. THE EXPERIMENTAL RESULTS

\begin{tabular}{|c|c|c|}
\hline The number & Artificial counting & Automatic counting \\
\hline 1 & 50 & 0050 \\
\hline 2 & 150 & 0150 \\
\hline 3 & 350 & 0350 \\
\hline 4 & 550 & 0550 \\
\hline 5 & 1050 & 1050 \\
\hline
\end{tabular}

The above results show that the counting system with AT89C51 used as the control center and optoelectronic switch used as the sensor has the advantages of simple structure, low cost . According to the test, the device is stable and reliable, has not found the misoperation, and can be widely used quantity statistics in storage, production line and so on.

\section{ACKNOWLEDGEMENTS}

The authors would like to express her gratitude to Professor Xiaofeng $\mathrm{Wu}$ for his valuable discussions and suggestions. Thanks are also to all colleagues who previously provide technical support.

\section{REFERENCES}

[1] Hirotani Daisuke, Myreshka1, Morikawa Katsumi1and Takahashi Katsuhiko1, "Analysis and design of self-balancing production line,”Computers and Industrial Engineering , vol.50 , August 2006 , pp. 488-502,doi:10.1016/j.cie.2005.06.008.

[2] Pornpanomchai, Chomtip,Stheitsthienchai, Fuangchat, Rattanachuen and Sorawat,“Object detection and counting system,”1st International Congress on Image and Signal Processing, (CISP 2008) September 2008,vol. 2, pp. 61-65, doi:10.1109/CISP.2008.108.

[3] Lijun Feng, "The design and making of the detecting,counting and displaying device on the assembly line",Journal of Foshan University(Natural Science Edition), October 2006, vol.24, pp. 35-37.

[4] Lin C.L., Miller A.B., Luttrell G.H.and Adel G.T., "Development of on line coal washability analysis using x-ray computed tomography," Coal Preparation, vol.21, April 2000, pp.19-21.

[5] Zheng Enxing and Tang Jing, "Design and realization of color character overlay based on AT89S51 and MC1337”, 2010 International Conference on Applied Mechanics and Mechanical Engineering(ICAMME2010),pp.809-81813,doi:10.4028/AMM.2932.809 .

[6] http://www.atmel.com/zh/cn/devices/AT89S51.aspx?tab=overview

[7] SUN Bo,CHEN Gang,WANG Er-zhi1and ZHANG Bing-yi, " Intelligent SoC Data Terminal Processor Based on Embedded Idea Instrument”, Technique and Sensor, January 2008, pp. 77-79.

[8] Zhang Songbo and Zhang Dongsheng, “ Application of real-time clock 12887 in embedded system “, Electrical Measurement \& Instrumentation, August 2004,vol.41,pp. 54-56.

[9] Han Cheng-Hao, Gao Xiao-Hong, Zhao Ding-Xuan, Chen Wei-Li1 and Yang-Jia, “ Development of meter-reading system building centralized controller in intelligent residential district”,2008 International Conference on Wireless Communications, Networking and Mobile Computing, doi:10.1109/WiCom.2008.1189D.

[10] Lin C.L.,Miller A.B., Luttrell G.H. Baojun Ma, "The application of non-contact strap scale in automatic ingredient system," Journal of 
Liaoning Institute of Technology On Social Science, January 2001, vol. 21, pp. 33-34.

[11] Son Byung-Rak, Shin. Seung-Chan, Kim Jung-Gyu, and Her YongSork, "Implementation of the real-time people counting system using wireless sensor networks"International Journal of Multimedia and Ubiquitous Engineering, 2007, vol. 2, pp. 63-80. 